\title{
Complications following regional anesthesia versus general anesthesia for the treatment of distal radius fractures
}

\author{
Ryan Lee ${ }^{1}$. Danny Lee ${ }^{2}$ - Pradip Ramamurti $^{1}$ - Safa Fassihi ${ }^{3}$. Jessica H. Heyer ${ }^{3}$. Monica Stadecker ${ }^{3}$. \\ Michael Webber $^{3} \cdot$ Alice Hughes $^{3} \cdot$ Rajeev Pandarinath ${ }^{3}$
}

Received: 13 January 2021 / Accepted: 13 May 2021 / Published online: 29 May 2021

○) Springer-Verlag GmbH Germany, part of Springer Nature 2021

\begin{abstract}
Purpose Open reduction and internal fixation (ORIF) are commonly utilized for the repair of distal radius fractures (DRF). While general anesthesia (GA) is typically administered for ORIF, recent studies have also demonstrated promising results with the usage of regional anesthesia (RA) in the surgical treatment of distal radius fractures. This study will compare complication rates between the use of RA versus GA for ORIF of DRFs.

Methods A multi-institutional surgical registry was utilized to identify patients who had undergone ORIF for DRFs from 2005 to 2018-these patients were stratified into GA and RA cohorts. Patients were matched utilizing coarsened-exact-matching (CEM) to compare postoperative outcomes and rates of 30-day complications were compared between the two cohorts. Results Upon CEM-matching, 1191 patients receiving RA were matched to 9250 patients who had received GA, with a multivariate imbalance measure (L1) statistic of $<0.001$. In the matched-cohort analysis, no significant differences were observed in rates of any complication (all $p \geq 0.083$ ). On multivariate regression analyses, RA was not associated with increased risk for any complication $(p=0.445)$, minor complications $(p=0.093)$, major complications $(p=0.758)$, unplanned reoperations $(p=0.355)$, unplanned readmissions $(p=0.799)$, or mortality $(p=0.579)$.

Conclusion With similar safety profiles, RA is a safe and reasonable alternative to GA when managing DRFs surgically. RA may be the preferred anesthetic technique for ORIF of DRFs in patients at high risk with GA, such as those with reactions to GA in the past or with significant cardiopulmonary risk factors.
\end{abstract}

Keywords Distal radius fracture $\cdot$ Regional anesthesia $\cdot$ Complications $\cdot$ General anesthesia $\cdot$ Peripheral nerve block

Work was done at George Washington School of Medicine and Health Sciences.

Ryan Lee

ryanlee@gwmail.gwu.edu

Danny Lee

danny.lee1@jhsmiami.org

1 The George Washington University School of Medicine and Health Sciences, The George Washington University in Washington, 2300 M St, Washington, DC 20037, USA

2 Department of Orthopaedic Surgery, University of Miami-Jackson Memorial Health System in Miami, Miami, FL, USA

3 Department of Orthopaedic Surgery, The George Washington University Hospital in Washington, Washington, DC, USA

\section{Introduction}

Distal radius fractures (DRF) are the most common long bone fracture and are typically caused by a fall on an outstretched hand [1]. DRFs account for up to $18 \%$ of all fractures in the elderly population and up to $20 \%$ of all fractures treated in an emergency department setting [1-3]. Open reduction and internal fixation (ORIF) have recently become a mainstay of treatment in these cases as outcomes are very favorable with respect to healing and articular congruency $[2,4]$. However, ORIF of DRFs are not without complications. With DRFs being one of the most common orthopaedic injuries, it is important to try and mitigate any other complications that could be prevented.

One way to try and minimize complications is by utilizing regional anesthesia (RA). RA has been shown to have beneficial effects including decreased perioperative opioid consumption, decreased length of stay (LOS), and decreased 
nausea in various orthopaedic procedures [5-8]. In addition, the use of RA in ORIF of DRFs has been reported to contribute to decreased pain and increased range of motion earlier in the post-operative period potentially expediting rehabilitation. [7] Although the use of RA and outcomes following DRF have been previously explored, only singleinstitution studies have been conducted [5, 7, 9-12]. To the author's knowledge, no study has been conducted with a large patient cohort analyzing outcomes following RA versus general anesthesia (GA) for ORIF of DRFs. This present study seeks to utilize the American College of Surgeons National Quality Improvement Program (ACS-NSQIP) database to compare outcomes following ORIF of DRFs with a large nationwide patient population to verify previous reports' support for the role of RA. In doing so, surgeons can help mitigate complications for a very common injury and can better counsel patients on which type of anesthesia to utilize for the procedure.

\section{Materials and methods}

\section{Patient selection}

The ACS-NSQIP database was queried to identify all patients who had undergone surgery for the treatment of distal radius fracture from 2005 to 2018 . Patients were selected based on their Current Procedure Terminology (CPT) codes, corresponding with CPT 25607, 25608, and 25609. Patients were then stratified into two cohorts based on the type of anesthesia they received: GA or RA. Patients receiving other methods of anesthesia, including epidural, spinal, and monitored anesthesia care/intravenous (MAC/IV) sedation, were excluded from this study. The ACS-NSQIP database classifies specific cases based on the primary method of anesthesia utilized. As such, if RA or epidural anesthesia was utilized with MAC or IV sedation, the primary method of anesthesia recorded in the ACS-NSQIP database would be classified as MAC/IV sedation. In addition, if RA was utilized at first but GA had to be utilized, then the primary method of anesthesia recorded would be GA. As such, the present study only compares ORIF cases for DRF in which RA was the sole method of anesthesia utilized versus GA. A total of 14,503 patients met inclusion criteria, of which 1348 (9.29\%) had received regional anesthesia and 13,135 $(90.71 \%)$ had received GA.

\section{Variables}

The ACS-NSQIP database contains over 150 variables for each patient, providing a comprehensive medical history and detailed reporting on postoperative complications. Demographic information was collected to better characterize this patient cohort; demographic variables included age, gender, race, and BMI, which was calculated with the given height $(\mathrm{cm})$ and weight $(\mathrm{kg})$ values. Preoperative comorbidities were also analyzed for differences between obesity classes, including smoking history, diabetes mellitus, dyspnea, ventilator dependence, chronic obstructive pulmonary disease (COPD), ascites, congestive heart failure (CHF), hypertension requiring medication management, acute renal failure, dialysis, disseminated cancer, open wounds/wound infections, chronic steroid use, significant weight loss of more than $10 \%$ of total body weight within the six-month preoperative period, hematologic disorders (i.e. hemophilias, coagulopathies, vitamin deficiencies), preoperative blood transfusions (within $72 \mathrm{~h}$ of the start of surgery), systemic sepsis, and functional dependence. Perioperative variables, such as ASA (American Society of Anesthesiologists) classification and type of anesthetic administered, were also included for analysis.

Medical complications occurring within the 30-postoperative period are reported by the ACS-NSQIP database, in addition to administrative parameters such as length of hospital stay and discharge information. This retrospective study included complications that were present in all available years of the ACS-NSQIP database to minimize the confounding effects of missing and incomplete data. The postoperative complications included in this study were superficial surgical site infections (SSI), deep SSI, organ/ space SSI, wound dehiscence, pneumonia, unplanned intubation, pulmonary embolisms, ventilator dependence ( $>48 \mathrm{~h}$ ), progressive renal insufficiency, acute renal failure, urinary tract infections (UTI), cerebrovascular accidents (CVA)/strokes, cardiac arrest requiring cardiopulmonary resuscitation, myocardial infarction, transfusions, deep venous thromboembolisms (DVT), systemic sepsis, septic shock, unplanned reoperations, unplanned readmissions, and mortality.

\section{Statistical analysis}

Patient demographic factors, preoperative comorbidities, and postoperative complication rates were analyzed for differences based on the primary anesthetic that they had received. Pearson's chi-squared tests and Fischer's exact tests (when expected cell sizes were less than 5 and variance could not be assumed to be normally distributed) were used to assess for differences in categorical variables, which are reported as number of subjects $(n)$ and incidence/prevalence rates (\%). All continuous variables, such as age and time parameters, are expressed as mean values with their respective standard deviations. All continuous variables were analyzed for differences in mean with one-way analyses of variance (ANOVA).

To control for differences in patient characteristics and comorbidities, the present study implements a widely 
accepted method called coarsened exact matching (CEM). CEM has been used previously in the literature with large patient cohorts and has been recognized as a superior method of matching to the ones most commonly used in large-scale studies, such as propensity score matching [13-17]. CEM temporarily coarsens the data to produce exact matches based on the entered variables, allowing for less statistical assumptions and model dependence when attempting to estimate the effect of a specific treatment (regional vs. general anesthesia in the present study). Patients receiving RA were matched to those receiving GA, dropping cases that were not matched exactly on the factors that were entered for controlling. Patients were matched based on age, gender, race, BMI, smoking status, diabetes mellitus, dyspnea, ventilator dependence, $\mathrm{COPD}$, ascites, $\mathrm{CHF}$, hypertension, acute renal failure, dialysis, disseminated cancer, open wounds/wound infections, chronic steroid use, significant weight loss, hematologic disorders, preoperative transfusions, sepsis, functional dependence, and ASA classification. This exact matching method ensures the inclusion of patients who are healthily comparable with the most similar baseline characteristics. These matched cohorts were all analyzed for differences in patient characteristics, as well as complication rates.

A multivariate logistic regression model was implemented with the weights provided by the CEM matching to analyze the independent effect of regional anesthesia on risks for complications in comparison to those receiving GA. Outcomes analyzed in the multivariate logistic regression models included any complication, minor complications, major complications, unplanned reoperation, unplanned readmission, and mortality. All statistical findings with $p$ values less than or equal to 0.05 were considered significant for this study. Variance was not assumed to be equal for any variable with the exception of age. All statistical analyses were performed using the IBM SPSS Version 25 Software (IBM Corp., Armonk, NY) and R๑ Version 3.3.3.

\section{Results}

A total of 14,503 patients met inclusion criteria, of which $1348(9.29 \%)$ had received RA and 13,135 (90.71\%) had received GA. In the unmatched analysis, the RA cohort was significantly older $(\bar{x}=59.82$ years, SD 15.805$)$ than the GA cohort $(\bar{x}=56.32$ years, SD 16.402; $p<0.001)$. The RA cohort was also comprised of a larger proportion of females (78.12 vs. $72.93 \% ; p<0.001)$ but a smaller proportion of obese patients (30.04 vs. $33.80 \%$; $p=0.016)$. Significant differences were also observed in the distribution of race/ ethnicity $(p<0.001)$. (Supplementary Table 1 ).

In the unmatched analysis, the RA cohort presented with significantly greater rates of disseminated cancer $(0.59$ vs. $0.18 \% ; p=0.008)$ and functional dependence (3.12 vs.
$1.89 \% ; p=0.001)$ than the GA cohort. However, the RA cohort presented with a smaller rate of smoking history (14.61 vs. $20.05 \% ; p<0.001)$. (Supplementary Table 1 ).

Upon CEM-matching, 1191 patients receiving RA were matched to 9250 patients who had received GA, with a multivariate imbalance measure (L1) statistic of $<0.001$, indicating adequate matching with minimal imbalance. There were no significant differences observed in any demographic factors (all $p \geq 0.691$ ) or preoperative comorbidities (all $p \geq 0.868$ ). (Table 1 ) No differences were observed in perioperative parameters, with the exception of total operative time. On average, the RA cohort experienced longer operative times ( $\bar{x}=81.68 \mathrm{~min}$, SD 39.248) than the GA cohort $(\bar{x}=77.24 \mathrm{~min}, \mathrm{SD} 45.505 ; p=0.001)$ (Table 2).

In the matched-cohort analysis, no significant differences were observed in rates of any complication (all $p \geq 0.083$; Table 3). On multivariate regression analyses, RA was not associated with increased risk for any complication $(p=0.445)$, minor complications $(p=0.093)$, major complications $(p=0.758)$, unplanned reoperations $(p=0.355)$, unplanned readmissions $(p=0.799)$, or mortality $(p=0.579)$. (Table 4)

\section{Discussion}

The use of anesthetic techniques has revolutionized medicine since its inception in the nineteenth century. GA provides intraoperative amnesia and has been used successfully in a plethora of both orthopaedic and non-orthopaedic procedures as it can be continuously administered with quick reversibility in the event of unexpected complications [18]. However, GA is typically inadequate on its own at providing local pain control in the immediate post-operative period and can suppress normal autonomic function requiring increased surveillance/monitoring [18]. Less common side effects of GA and intubation include, but are not limited to, aspiration, nausea, pruritis, and hoarseness [18]. In comparison to GA, RA avoids these aforementioned side effects and has been associated with improved postoperative pain control, decreased opioid use, and reduced recovery times in certain orthopedic procedures [18-22] RA with laryngeal mask airway has also been reported to be associated with decreased rates of intraoperative hemodynamic instability and postoperative cognitive function compared to GA with endotracheal intubation in elderly patients for intertrochanteric fracture management [23]. RA has demonstrated other advantages including preservation of autonomic function, improved muscle relaxation, avoidance of airway manipulation, decreased nausea/vomiting, and reduced post-anesthesia care unit stay $[18,24,25]$.

This current study demonstrates no significant differences in rates of any complications between the matched 
Table 1 Demographics and comorbidities in CEM-matched general vs. regional anesthesia

\begin{tabular}{|c|c|c|c|c|c|}
\hline \multirow{2}{*}{$\overline{\text { Demographics }}$} & \multicolumn{2}{|c|}{ General $(n=9250)$} & \multicolumn{2}{|c|}{ Regional $(n=1191)$} & \multirow[t]{2}{*}{$P$ value } \\
\hline & & & & & \\
\hline Age $(\text { Mean } \pm S D)^{\mathrm{a}}$ & $55.68 \pm 16.738$ & & $55.88 \pm 16.811$ & & 0.691 \\
\hline Age (years) & & & & & 1.000 \\
\hline$x \leq 40$ & 1906 & $20.61 \%$ & 245 & $20.57 \%$ & \\
\hline $40<x \leq 50$ & 1006 & $10.88 \%$ & 130 & $10.92 \%$ & \\
\hline $50<x \leq 60$ & 2249 & $24.31 \%$ & 290 & $24.35 \%$ & \\
\hline $60<x \leq 70$ & 2408 & $26.03 \%$ & 310 & $26.03 \%$ & \\
\hline $70<x$ & 1681 & $18.17 \%$ & 216 & $18.14 \%$ & \\
\hline Sex & & & & & 0.976 \\
\hline Female & 7079 & $76.53 \%$ & 911 & $76.49 \%$ & \\
\hline Male & 2171 & $23.47 \%$ & 280 & $23.51 \%$ & \\
\hline Race/Ethnicity & & & & & 0.997 \\
\hline American Indian or Alaska Native & 0 & $0.00 \%$ & 0 & $0.00 \%$ & \\
\hline Asian or Pacific Islander & 222 & $2.40 \%$ & 29 & $2.43 \%$ & \\
\hline Black or African American & 102 & $1.10 \%$ & 13 & $1.09 \%$ & \\
\hline Hispanic & 0 & $0.00 \%$ & 0 & $0.00 \%$ & \\
\hline White or Caucasian & 8926 & $96.50 \%$ & 1149 & $96.47 \%$ & \\
\hline Body mass index $\left(\mathrm{kg} / \mathrm{m}^{2}\right)$ & & & & & 1.000 \\
\hline Normal & 3432 & $37.10 \%$ & 442 & $37.11 \%$ & \\
\hline Overweight & 3300 & $35.68 \%$ & 425 & $35.68 \%$ & \\
\hline Class I obese & 1643 & $17.76 \%$ & 212 & $17.80 \%$ & \\
\hline Class II obese & 544 & $5.88 \%$ & 70 & $5.88 \%$ & \\
\hline Class III obese & 331 & $3.58 \%$ & 43 & $3.61 \%$ & \\
\hline \multicolumn{6}{|l|}{ Pre-operative comorbidities } \\
\hline Diabetes mellitus & & & & & 0.996 \\
\hline No diabetes mellitus & 9008 & $97.38 \%$ & 1160 & $97.40 \%$ & \\
\hline Non-insulin dependent & 189 & $2.04 \%$ & 24 & $2.02 \%$ & \\
\hline Insulin dependent & 53 & $0.57 \%$ & 7 & $0.59 \%$ & \\
\hline Smoking history & 1420 & $15.35 \%$ & 183 & $15.37 \%$ & 0.990 \\
\hline Dyspnea & & & & & 0.934 \\
\hline No dyspnea & 9227 & $99.75 \%$ & 1188 & $99.75 \%$ & \\
\hline Moderate exertion & 22 & $0.24 \%$ & 3 & $0.25 \%$ & \\
\hline At rest & 1 & $0.01 \%$ & 0 & $0.00 \%$ & \\
\hline Ventilator dependence & 0 & $0.00 \%$ & 0 & $0.00 \%$ & - \\
\hline COPD & 71 & $0.77 \%$ & 9 & $0.76 \%$ & 0.965 \\
\hline Ascites & 0 & $0.00 \%$ & 0 & $0.00 \%$ & - \\
\hline Congestive heart failure & 1 & $0.01 \%$ & 0 & $0.00 \%$ & 1.000 \\
\hline Hypertension & 2572 & $27.81 \%$ & 331 & $27.79 \%$ & 0.992 \\
\hline acute renal failure & 0 & $0.00 \%$ & 0 & $0.00 \%$ & - \\
\hline Dialysis & 0 & $0.00 \%$ & 0 & $0.00 \%$ & - \\
\hline Disseminated cancer & 0 & $0.00 \%$ & 0 & $0.00 \%$ & - \\
\hline Open wounds/wound infections & 15 & $0.16 \%$ & 2 & $0.17 \%$ & 1.000 \\
\hline Chronic steroid use & 32 & $0.35 \%$ & 4 & $0.34 \%$ & 1.000 \\
\hline Weight loss & 0 & $0.00 \%$ & 0 & $0.00 \%$ & - \\
\hline Bleeding disorders & 39 & $0.42 \%$ & 5 & $0.42 \%$ & 0.993 \\
\hline Transfusions (within $72 \mathrm{~h}$ preop) & 0 & $0.00 \%$ & 0 & $0.00 \%$ & - \\
\hline Systemic sepsis & 1 & $0.01 \%$ & 0 & $0.00 \%$ & 1.000 \\
\hline Functional status & & & & & 0.868 \\
\hline Independent & 9208 & $99.55 \%$ & 1186 & $99.58 \%$ & \\
\hline Partially dependent & 42 & $0.45 \%$ & 5 & $0.42 \%$ & \\
\hline Totally dependent & 0 & $0.00 \%$ & 0 & $0.00 \%$ & \\
\hline
\end{tabular}


Table 1 (continued)

\begin{tabular}{lllll}
\hline & General $(n=9250)$ & Regional $(n=1191)$ & $P$ value \\
\hline ASA classification & & & & 0.996 \\
1-No disturb & 1700 & $18.38 \%$ & 219 & $18.39 \%$ \\
2-Mild disturb & 5796 & $62.66 \%$ & 746 & $62.64 \%$ \\
3-Severe disturb & 1744 & $18.85 \%$ & 225 & $18.89 \%$ \\
4-Life threat & 10 & $0.11 \%$ & 1 & $0.08 \%$ \\
5- Moribund & 0 & $0.00 \%$ & 0 & $0.00 \%$ \\
None & 0 & $0.00 \%$ & 0 & $0.00 \%$ \\
\hline
\end{tabular}

All other values expressed as (\%) and $N$

COPD Chronic obstructive pulmonary disease

${ }^{\mathrm{a}}$ Values expressed as Mean \pm Standard Deviation (SD)

Table 2 Perioperative parameters in CEM-matched general vs. regional anesthesia cohorts

\begin{tabular}{|c|c|c|c|c|c|}
\hline & General $(n=9250$ & & Regional $(n=1191)$ & & $P$ value \\
\hline Days to operation from admission & $0.22 \pm 6.258$ & & $0.06 \pm 0.774$ & & 0.377 \\
\hline Total operating time (Minutes) & $77.24 \pm 45.505$ & & $81.68 \pm 39.248$ & & 0.001 \\
\hline Total hospital stay length (Days) & $0.52 \pm 5.847$ & & $0.34 \pm 2.213$ & & 0.304 \\
\hline Days from operation to discharge & $0.33 \pm 2.065$ & & $0.28 \pm 2.035$ & & 0.463 \\
\hline Wound classification & & & & & 0.084 \\
\hline 1-Clean & 9018 & $97.49 \%$ & 1174 & $98.57 \%$ & \\
\hline 2-Clean/contaminated & 137 & $1.48 \%$ & 11 & $0.92 \%$ & \\
\hline 3-Contaminated & 69 & $0.75 \%$ & 6 & $0.50 \%$ & \\
\hline 4-Dirty/infected & 26 & $0.28 \%$ & 0 & $0.00 \%$ & \\
\hline
\end{tabular}

RA and GA cohorts. The lack of differences in complication rates implicates that RA is a reasonable and safe alternative to GA for ORIF of DRFs. RA has been demonstrated to be efficacious and safe in other orthopaedic procedures as well. Helwani et al. previously reported reductions in post-operative deep SSI rates, hospital LOS, and major complications in patients undergoing total hip arthroplasty when utilizing RA compared to GA [8]. Liu et al. similarly reports a reduced risk of pneumonia and systemic infectious complications following total knee arthroplasty with neuraxial anesthesia compared to GA [23]. While the present study did not demonstrate a reduction in these adverse events and did not analyze the effects of neuraxial anesthesia, no significant differences were exhibited in terms of any complications, unplanned reoperations, or morality when comparing RA and GA for ORIF of DRFs. The lack of differences in adverse events demonstrated in this study further supports the safety profile of RA and its role as a reasonable alternative to GA in ORIF of DRFs. Certain patient demographics and comorbidities, such as obesity, greater age, and frailty, are known to increase the risk of adverse events with GA [26]. For example, GA has been associated with an increased risk of postoperative cognitive dysfunction in elderly patients when compared to other forms of anesthesia [27]. The current findings suggest that, without any differences in complication rates, RA for DRF is a reasonable/safe alternative, and may be the preferred mode of anesthesia for high-risk patients-future work exploring the safety profile of RA in ORIF of DRFs in these high-risk patients is warranted. However, as with all other aspects of a patient's care, the options and alternatives of anesthesia available should be thoroughly discussed with the patient so that factors such as anxiety and comfort can be taken into account. In doing so, the patient can make an informed decision with the perioperative care team's recommendations.

There is currently debate within the literature as to whether RA improves functional outcomes following ORIF of DRFs in comparison to GA. However, brachial plexus blockade in upper extremity surgery has generally demonstrated reduced systemic anesthetic requirements, lower perioperative opioid consumption, decreased nausea, and shortened postoperative stays $[5,7,8,28,29]$. In a randomized control trial, Hadzic et al. reported lower pain scores for patients undergoing hand/wrist surgery who had received RA compared to GA in the perioperative period [30]. Egol et al. reported significantly decreased pain and improved function at three and six-month follow-up in patients who had received RA as opposed to GA for ORIF of DRF [7]. However, pain and functional scores equalized at 12-month follow-up between the two cohorts [7]. In an analysis of elderly patients who required surgery for DRF, Tabrizi et al. compared the use of hematoma blocks versus GA and found 
Table 3 Postoperative complication rates in CEM-matched general vs. regional anesthesia cohorts

\begin{tabular}{|c|c|c|c|c|c|}
\hline \multirow[t]{2}{*}{ Postoperative complications } & \multicolumn{2}{|c|}{$\begin{array}{l}\text { General } \\
(n=9250)\end{array}$} & \multicolumn{2}{|c|}{$\begin{array}{l}\text { Regional } \\
(n=1191)\end{array}$} & \multirow[t]{2}{*}{$P$ value } \\
\hline & 9250 & & 119 & & \\
\hline Any complication & 187 & $2.02 \%$ & 28 & $2.35 \%$ & 0.451 \\
\hline Minor complication & 44 & $0.48 \%$ & 10 & $0.84 \%$ & 0.127 \\
\hline Superficial incisional SSI & 11 & $0.12 \%$ & 4 & $0.34 \%$ & 0.083 \\
\hline Wound disruption & 2 & $0.02 \%$ & 1 & $0.08 \%$ & 0.305 \\
\hline Pneumonia & 6 & $0.06 \%$ & 0 & $0.00 \%$ & 1.000 \\
\hline Progressive renal insufficiency & 1 & $0.01 \%$ & 0 & $0.00 \%$ & 1.000 \\
\hline Urinary tract infection & 15 & $0.16 \%$ & 4 & $0.34 \%$ & 0.264 \\
\hline Blood transfusions & 9 & $0.10 \%$ & 1 & $0.08 \%$ & 1.000 \\
\hline Major complication & 151 & $1.63 \%$ & 18 & $1.51 \%$ & 0.755 \\
\hline Deep incisional SSI & 6 & $0.06 \%$ & 0 & $0.00 \%$ & 1.000 \\
\hline Organ/space SSI & 2 & $0.02 \%$ & 0 & $0.00 \%$ & 1.000 \\
\hline Unplanned intubation & 4 & $0.04 \%$ & 0 & $0.00 \%$ & 1.000 \\
\hline Pulmonary embolism & 3 & $0.03 \%$ & 0 & $0.00 \%$ & 1.000 \\
\hline $\begin{array}{l}\text { Ventilator dependence } \\
(>48 \mathrm{~h})\end{array}$ & 0 & $0.00 \%$ & 0 & $0.00 \%$ & - \\
\hline Acute renal failure & 0 & $0.00 \%$ & 0 & $0.00 \%$ & - \\
\hline CVA/stroke & 3 & $0.03 \%$ & 2 & $0.17 \%$ & 0.103 \\
\hline Cardiac arrest & 2 & $0.02 \%$ & 0 & $0.00 \%$ & 1.000 \\
\hline Myocardial infarction & 1 & $0.01 \%$ & 1 & $0.08 \%$ & 0.215 \\
\hline DVT & 1 & $0.01 \%$ & 0 & $0.00 \%$ & 1.000 \\
\hline Systemic sepsis & 2 & $0.02 \%$ & 0 & $0.00 \%$ & 1.000 \\
\hline Septic shock & 0 & $0.00 \%$ & 0 & $0.00 \%$ & - \\
\hline Unplanned reoperation & 85 & $0.92 \%$ & 8 & $0.67 \%$ & 0.393 \\
\hline Unplanned readmission & 81 & $0.88 \%$ & 11 & $0.92 \%$ & 0.868 \\
\hline Mortality & 5 & $0.05 \%$ & 1 & $0.08 \%$ & 0.517 \\
\hline
\end{tabular}

SSI Surgical Site Infection, CVA Cerebral Vascular Accident, DVT Deep Venous Thromboembolism

Table 4 Multivariate logistic regression model analyzing regional anesthesia as a risk factor for complications

\begin{tabular}{lllll}
\hline Postoperative complications & OR & $95 \%$ CI & $P$ value \\
\hline Any complication & 1.170 & 0.782 & 1.751 & 0.445 \\
Minor complication & 1.803 & 0.907 & 3.587 & 0.093 \\
Major complication & 0.925 & 0.565 & 1.516 & 0.758 \\
Unplanned reoperation & 0.706 & 0.337 & 1.477 & 0.355 \\
Unplanned readmission & 1.085 & 0.580 & 2.030 & 0.799 \\
Mortality & 1.789 & 0.230 & 13.912 & 0.579 \\
\hline
\end{tabular}

similar results-patients who were given the hematoma block demonstrated significantly decreased pain intensity in the initial hours following fracture reduction and had reduced rates of intensive care unit admissions [12]. The present study, however, was unable to analyze functional outcome and pain scores at long-term follow-up as the ACSNSQIP database does not record this information. The lack of long-term data and functional outcomes thus represents a limitation inherent to the database.

Although RA may avoid the risks of GA and provide adequate pain control in the immediate postoperative period, rebound pain is a known phenomenon associated with RA following various orthopaedic procedures [5, 31, 32]. Galos et al. reports no differences in adverse events between patients who had received RA versus GA in the treatment of DRF [5]. However, the authors demonstrate that although GA is associated with greater immediate postoperative pain, peripheral nerve blocks are often associated with delayed rebound pain in the postoperative period [5]. In a retrospective quality improvement analysis, Sunderland et al. report increased rates of unplanned physician office visits following ORIF of DRFs when utilizing RA due to delayed rebound pain compared to GA [10]. As such, although RA may provide benefits by avoiding risks associated with GA in high-risk patient populations, thorough postoperative/discharge analgesic medications should be implemented to curb rebound pain. As the ACS-NSQIP database does not record pain scores, the lack of data regarding patient pain levels is another limitation inherent to this study when comparing RA and GA for operative outcomes of DRFs.

Regarding peri-operative variables, the RA group had shorter days to operation from admission, total hospital stay length, and days from operation to discharge-however, these results were not statistically significant. Of note, RA had a significantly longer operative time $(81.68 \mathrm{~min})$ compared to GA (77.24 min). The cause for a longer operative time in the RA group is most likely multifactorial. Although the present study was able to control for differences in patient characteristics/comorbidities via CEM, only the variables/comorbidities that are maintained by the ACSNSQIP database were controlled for. As this is a nationwide database, the granularity of information provided is limited. As such, the longer operative time in the RA group may be due to a sicker/more complex patient population that opted for RA who had variables that were unable to be controlled for (such as different grades of congestive heart failure or ascites). It is plausible that if RA was used for sicker/more complex patients, a longer operative time would be expected.

There are several additional limitations to this retrospective study. A limitation inherent to this study is the granularity of information provided by the ACS-NSQIP database. Although the database records the main mode of anesthetic utilized as discussed in the materials and methods, there is no data available as to what or if secondary/supplemental modes of anesthesia were utilized. As such, this represents a potential confounding variable that this study could not account for. In addition, the ACS-NSQIP database does not differentiate between types of RA, such as single-shot or continuous infusion of anesthetics. This could thus represent a systemic confounding variable that this study could 
not account for and could potentially influence the overall complication rates. Furthermore, another confounding variable that could not be accounted for is the type of injury in each DRF. As the ACS-NSQIP database does not record the type of DRFs of each patient, further work comparing complication rates following ORIF of DRF between RA and GA in specific types of DRFs are needed. Inherent to all large database studies, CPT codes were originally designed for insurance purposes rather than for research. As such, there may be skewing of data based on financial incentive [33]. Finally, as the ACS-NSQIP database is a large multiinstitutional surgical registry, there is currently no way of verifying the accuracy of the data inputted.

\section{Conclusion}

The present study demonstrated no differences in adverse events/complications between RA and GA for ORIF of DRFs. With similar safety profiles, RA is a safe and reasonable alternative to GA when managing DRFs surgically. RA may be the preferred anesthetic technique for ORIF of DRFs in patients at high risk with GA. However, the current literature indicates an increase in instances of rebound pain when utilizing RA following ORIF of DRFs that this study could not comment on. Proper analgesic protocols must be put in place for postoperative/discharge planning in anticipation of delayed rebound pain with the use of RA to adequately control patients' pain.

Supplementary Information The online version contains supplementary material available at https://doi.org/10.1007/s00068-021-01704-1.

Funding No benefits in any form have been received or will be received from a commercial party related directly or indirectly to the subject of this article.

Data availability Publicly available through the American College of Surgeons National Surgical Quality Improvement Program Database.

\section{Declarations}

Conflict of interest The authors declare that they have no conflict of interest.

\section{References}

1. MacIntyre NJ, Dewan N. Epidemiology of distal radius fractures and factors predicting risk and prognosis. $\mathrm{J}$ Hand Ther. 2016;29(2):136-45.

2. Meena S, Sharma P, Sambharia A, et al. Fractures of distal radius: an overview. J Fam Med Prim Care. 2014;3(4):325.

3. Nellans KW, Kowalski E, Chung KC. The epidemiology of distal radius fractures. Hand Clin. 2012;28(2):113-25.
4. Porter M, Stockley I. Fractures of the distal radius: intermediate and end results in relation to radiologic parameters. Clin Orthop Relat Res. 1987;220:241-52.

5. Galos DK, Taormina DP, Crespo A, et al. Does brachial plexus blockade result in improved pain scores after distal radius fracture fixation? a randomized trial. Clin Orthop Relat Res. 2016;474(5):1255-6.

6. Chen HP, Shen SJ, Tsai HI, Kao SC, Yu HP. Effects of interscalene nerve block for postoperative pain management in patients after shoulder surgery. Biomed Res Int. 2015;2015:1-5.

7. Egol KA, Soojian MG, Walsh M, et al. Regional anesthesia improves outcome after distal radius fracture fixation over general anesthesia. J Orthop Trauma. 2012;26(9):545-9.

8. Helwani MA, Avidan MS, Ben Abdallah A, et al. Effects of regional versus general anesthesia on outcomes after total hip arthroplasty. J Bone Jt Surgery-American. 2015;97(3):186-93.

9. Rundgren J, Mellstrand Navarro C, Ponzer S, et al. Regional or general anesthesia in the surgical treatment of distal radial fractures: a randomized clinical trial. J Bone Jt Surg - Am. 2019;101(13):1168-76.

10. Sunderland S, Yarnold $\mathrm{CH}$, Head SJ, et al. Regional versus general anesthesia and the incidence of unplanned health care resource utilization for postoperative pain after wrist fracture surgery: results from a retrospective quality improvement project. Reg Anesth Pain Med. 2016;41(1):22-7.

11. O'Neil JT, Wang ML, Kim N, et al. Prospective evaluation of opioid consumption after distal radius fracture repair surgery. Am J Orthop (Belle Mead NJ). 2017;46(1):E35-40.

12. Tabrizi A, Tolouei FM, Hassani E, et al. Hematoma block versus general anesthesia in distal radius fractures in patients over 60 years in trauma emergency. Anesthesiol Pain Med. 2017;7(1):1-5.

13. Ottesen TD, Zogg CK, Haynes MS, et al. Dialysis patients undergoing total knee arthroplasty have significantly increased odds of perioperative adverse events independent of demographic and comorbidity factors. J Arthroplasty. 2018;33(9):2827-34.

14. Wilson JM, Farley KX, Bradbury TL, et al. Is spinal anesthesia safer than general anesthesia for patients undergoing revision THA? Analysis of the ACS-NSQIP Database. Clin Orthop Relat Res. 2020;478(1):80-7.

15. Azin A, Hirpara D, Jackson T, et al. Emergency laparoscopic and open repair of incarcerated ventral hernias: a multi-institutional comparative analysis with coarsened exact matching. Surg Endosc. 2019;33(9):2812-20.

16. Iacus SM, King G, Porro G. Multivariate matching methods that are monotonic imbalance bounding. J Am Stat Assoc. 2011;106(493):345-61.

17. Iacus SM, King G, Porro G. Causal inference without balance checking: coarsened exact matching. Polit Anal. 2012;20(1):1-24.

18. Ding DY, Mahure SA, Mollon B, et al. Comparison of general versus isolated regional anesthesia in total shoulder arthroplasty: a retrospective propensity-matched cohort analysis. J Orthop. 2017; 14(4):417-24.

19. Herrick MD, Liu H, Davis M, et al. Regional anesthesia decreases complications and resource utilization in shoulder arthroplasty patients. Acta Anaesthesiol Scand. 2018;62(4):540-7.

20. Wang H, Ma L, Yang D, et al. Cervical plexus anesthesia versus general anesthesia for anterior cervical discectomy and fusion surgery: a randomized clinical trial. Med (United States). 2017;96(7):e6119. https://doi.org/10.1097/MD.0000000000 006119.

21. Mirza F, Brown AR. Ultrasound-guided regional anesthesia for procedures of the upper extremity. Anesthesiol Res Pract. 2011. https://doi.org/10.1155/2011/579824.

22. Lee HY, Kim SH, So KY, et al. Effects of interscalene brachial plexus block to intra-operative hemodynamics and postoperative 
pain for arthroscopic shoulder surgery. Korean $\mathbf{J}$ Anesthesiol. 2012;62(1):30-4.

23. Liu J, Chenjuan M, Elkassabany N, et al. Neuraxial anesthesia decreases post-operative systemic infection risk compared to general anesthesia in knee arthroplasty. Anesth Analg. 2014;117(4):1010-6.

24. Ganta A, Ding D, Fisher N, et al. Continuous infraclavicular brachial block versus single-shot nerve block for distal radius surgery: a prospective randomized control trial. J Orthop Trauma. 2018;32(1):22-6.

25. Richman JM, Liu SS, Courpas G, et al. Does continuous peripheral nerve block provide superior pain control to opioids? A metaanalysis Anesth Analg. 2006;102(1):248-57.

26. Walsh MT. Improving outcomes in ambulatory anesthesia by identifying high risk patients. Curr Opin Anaesthesiol. 2018;31(6):659-66.

27. Mason SE, Noel-Storr A, Ritchie CW. The impact of general and regional anesthesia on the incidence of post-operative cognitive dysfunction and post-operative delirium: a systematic review with meta-analysis. J Alzheimer's Dis. 2010. https://doi.org/10.3233/ JAD-2010-101086.
28. Chelly JE, Greger J, Al Samsam T, et al. Reduction of operating and recovery room times and overnight hospital stays with interscalene blocks as sole anesthetic technique for rotator cuff surgery. Minerva Anestesiol. 2001;67(9):613-9.

29. Mariano ER, Sandhu NS, Loland VJ, et al. A randomized comparison of infraclavicular and supraclavicular continuous peripheral nerve blocks for postoperative analgesia. Reg Anesth Pain Med. 2011;36(1):26-31.

30. Hadzic A, Arliss J, Yufa M, et al. A comparison of infraclavicular nerve block. Anesthesiology. 2004;1:127-32.

31. Williams B, Bottegal M, Kentor M, et al. Rebound pain scores as a function of femoral nerve block duration after anterior cruciate ligament reconstruction: retrospective analysis of a prospective, randomized clinical trial. Reg Anesth Pain Med. 2007;32(3):186-92.

32. Goldstein RY, Montero N, Jain SK, et al. Efficacy of popliteal block in postoperative pain control after ankle. J Orthop Trauma. 2012;26(10):557-62.

33. Yoshihara H, Yoneoka D. Understanding the statistics and limitations of large database analyses. Spine (Phila Pa 1976). 2014;39(16):1311-2. 